\title{
THE CHANGES WHICH ALTER RENAL OSMOTIC WORK
}

\author{
By J. DAVID NEWBURGH
}

\author{
(Ann Arbor, Michigan)
}

(Received for publication December 28, 1942)

\section{INTRODUCTION}

Although realization that the kidney must do osmotic work antedates the greater part of our present knowledge of renal function, the implications of this requirement have not received the consideration they deserve, either by physiologists or by clinicians. Physiologically, there is, for instance, in terms of osmotic work, an optimal urine volume for removal of a given quantity and assortment of substances in urine. Clinically, there is the consideration of the consequences of the inability on the part of disabled kidneys to do sufficient osmotic work. Since, by adjustments of food and water intake, the kidneys' task can be changed in directions which will reduce osmotic work, regard for osmotic capacity is obviously of therapeutic importance. Reduction of the amount of a substance ingested does not necessarily reduce the osmotic work done by the kidney.

von Rhorer (1), in 1905, obtained an equation for the work required to excrete a single urinary component and this equation has been used by Addis (2) in studying experimental nephritis. In the present paper, it is undertaken to derive and examine the equation which will evaluate the osmotic work required for the simultaneous removal or non-removal of several substances and which will permit estimation of the adjustments that will produce reduced osmotic work.

\section{DERIVATION OF THE BASIC EQUATIONS}

The osmotic work of the kidney is done in converting the glomerular filtrate into urine. Inasmuch as this is an isothermal process, the reversible or minimal work required will be only a function of the initial and final states, and thus the work calculated over any convenient reversible path will equal the work for any other reversible path. This is illustrated by the fact that the equation giving the amount of work may be derived by considering several different hypothetical processes resulting in the formation of urine.
The equation may be derived by an extension of von Rhorer's method. A theoretical justification of the equation, as used by Barcroft, has been given by R. G. Dickinson and R. C. Tolman in an article by Borsook and Winegarden (3). The following derivation employs only one of several convenient paths which suggest themselves. We hypothesize the following simple process. One liter of glomerular filtrate is considered to form one liter of urine by the addition of certain substances to it, and the removal of other substances by the tubules. ${ }^{1}$

The amount of work $d E$ required to produce an infinitesimal change in volume $d v$ against a pressure $p$ is given by

$$
d E=-p d v .
$$

By van't Hoff's law we have $p=c R T$, where $c$ is the osmolar concentration, $R$ is the gas constant, and $T$ is the absolute temperature. Also, for one osmol

therefore

$$
v=\frac{1}{c} \quad \text { and } \quad d v=-\frac{1}{c^{2}} d c,
$$

$$
d E=-p d v=c R T \frac{1}{c^{2}} d c=R T \frac{d c}{c}
$$

The amount of energy required for the reversible transfer of one mol of substance from a solution of constant concentration $a$ to concentration $b$ will then be ${ }^{2}$

$$
E=\int_{a}^{b} R T \frac{d c}{c}=R T \int_{a}^{b} \frac{d c}{c}=R T \ln \frac{b}{a},
$$

where $\ln$ is the logarithm to the base $e$.

1 Clearly, one liter of urine is not derived from one liter of glomerular filtrate in fact. However, since the process considered here and the actual process both accomplish the formation of urine from a solution having the composition of extracellular fluid, the hypothetical process is a valid basis for the equations, regardless of the enormous volume of the actual glomerular filtrate.

2 Two very large vessels at temperature $T$, with regions permeable only to the solute in question are imagined. This solute is allowed to diffuse reversibly into a cylinder 
The energy needed to transfer an infinitesimal amount of substance, $d z_{i}$, from a reservoir of constant concentration $c_{i}$ with respect to the $i$ th component to a one liter volume of concentration $z_{i}$ will be given, according to equation (1) above, as

$$
d E=R T \ln \frac{z_{i}}{c_{i}} d z_{i}
$$

Integration of this between the limits $c_{i}$ and $x_{i}$, the urinary concentration of the $i$ th component, will correspond to the energy, with regard to the $i$ th component, required to form one liter of urine: ${ }^{3}$

$$
\begin{array}{r}
E_{i}=\int_{c_{i}}^{x_{i}} R T \ln \frac{z_{i}}{c_{i}} d z_{i}=c_{i} R T \int \ln \frac{z_{i}}{c_{i}} d\left(\frac{z_{i}}{c_{i}}\right) \\
=c_{i} R T\left[\frac{z_{i}}{c_{i}}\left(\ln \frac{z_{i}}{c_{i}}-1\right)\right]^{z_{i}=x_{i}} \\
=R T\left(x_{i} \ln \frac{x_{i}}{c_{i}}+c_{i}-x_{i}\right) .
\end{array}
$$

If there are $n$ liters of urine in a given time, and $y_{i}=n x_{i}$ is the corresponding total number of osmols of the $i$ th component, the total amount of work will be, for that component,

$$
\begin{aligned}
n E_{i}=n R T\left(x_{i}\right. & \left.\ln \frac{x_{i}}{c_{i}}+c_{i}-x_{i}\right) \\
& =R T y_{i}\left(\ln \frac{x_{i}}{c_{i}}+\frac{c_{i}}{x_{i}}-1\right)
\end{aligned}
$$

or

$$
\begin{aligned}
n E_{i} & =n R T\left(x_{i} \ln \frac{x_{i}}{c_{i}}+c_{i}-x_{i}\right) \\
& =R T\left(y_{i} \ln \frac{y_{i}}{n c_{i}}+n c_{i}-y_{i}\right) \\
& =R T\left(y_{i} \ln \frac{y_{i}}{c_{i}}-y_{i} \ln n+n c_{i}-y_{i}\right) .
\end{aligned}
$$

The total work $E_{T}$ done by the kidney will be the sum of the energies for the individual com-

from the first vessel at partial pressure $a$; a piston then compresses the substance to pressure $b$, and it is then allowed to diffuse into the other vessel at a pressure $b$. This is a simple application of one of the ideas involved in the "equilibrium box." A discussion of this and van't Hoff's law may be found in texts on physical chemistry, for example, Glasstone's "Text-Book of Physical Chemistry."

'See, for example, Dwight's "Table of Integrals." ponents:

$$
\begin{aligned}
E_{T}= & \sum_{i} n E_{i} \\
= & \sum_{i} R T\left(y_{i} \ln \frac{y_{i}}{c_{i}}-y_{i} \ln n+n c_{i}-y_{i}\right) \\
= & R T\left(\sum_{i} y_{i} \ln \frac{y_{i}}{c_{i}}\right. \\
& \left.\quad-\ln n \sum_{i} y_{i}+n \sum_{i} c_{i}-\sum_{i} y_{i}\right) .
\end{aligned}
$$

These equations hold accurately only for dilute solutions; for more concentrated solutions, the concentrations must be replaced by activities. This would, however, obscure the simplicity of the results, and in many cases, sufficient data are not at hand to make this change. Quantitative results of these equations will be only slightly inaccurate, and for most biological purposes may be considered valid.

The values of the energy given above are the values required for the idealized, thermodynamically reversible formation of urine, and thus represent the useful work done by the kidney, rather than the actual energy it must expend. Both inefficiency in the actual performance of the osmotic work, and simple back diffusion, will reduce the overall efficiency of the tubules.

\section{ON THE REDUCTION OF OSMOTIC WORK}

A time-honored form of treatment for any diseased organ is the reduction of the work that must be done by that organ. Applied to the kidney this implies the reduction of the osmotic work. Equation (4) above should thus be examined with the view of making $E_{T}$ as small as possible. One important and easily controlled variable is the total volume $n$ of the urine, determined largely by the amount of water drunk. Considering the rate of excretion $y_{i}$ of the various components, and also the composition of the glomerular filtrate as fixed, we may find the value of $n$ to make $E_{r}$ a minimum by setting

$$
\frac{\partial E_{T}}{\partial n}=0 \text {. }
$$

From equation (4) this gives

$$
\frac{\partial E_{T}}{\partial n}=R T\left(-\frac{1}{n} \sum_{i} y_{i}+\sum_{i} c_{i}\right)=0
$$


whence

$$
\sum_{i} c_{i}=\frac{1}{n} \sum_{i} y_{i}=\sum_{i} x_{i}
$$

Thus we see that to make the total work the least, the amount of water should be adjusted so that the total osmolar concentration of the urine $\left(\sum x_{i}\right)$ equals the total osmolar concentration of the glomerular filtrate $\left(\sum c_{i}\right)$. For a human urine of ordinary composition, the work is plotted as a function of the total daily volume in Figure 1, curve $A$. The minimal value is seen to be at $n=2.36$ liters, in agreement with equation (5): Although urea is the main urinary constituent, $E_{T}$ has its minimum at a point where the ratio of $x_{\text {urea }}$ to $c_{\text {urea }}$ is still large, due to the fact that there are constituents with a ratio $\frac{x_{i}}{c_{i}}$ much less than 1 (almost 0 for glucose), and for these the work increases with increasing $\boldsymbol{n}$.

It is interesting to note that the usual urine volume is not far from the volume which results in the minimal work. Due to the small slope of the curve in the region of the minimum, the amount of work associated with the ordinary urine volume is even more strikingly close to the minimal work.

Although not as easily subjected to control as the volume $n$, changes in the rate of excretion of the solid components are important. Two cases may be distinguished, one in which $\boldsymbol{n}$ is constant and only the amount of one solid changes, and the other in which $n$ is always adjusted so as to be at the optimal value. The first case is solved directly from equation (4), and the second case from equation (4) after the value of $n$ given by equation (5) has been substituted in (4). Fixing our attention on the $j$ th component, we want to determine $y_{j}$ so as to make $E_{T}$ a minimum, and so we set

$$
\frac{\partial E_{T}}{\partial y_{j}}=0 .
$$

In the first case this gives

$$
\frac{\partial E_{T}}{\partial y_{j}}=R T\left(y_{j} \frac{c_{j}}{y_{j}} \frac{1}{c_{j}}+\ln \frac{y_{j}}{c_{j}}-\ln n-1\right)=0,
$$

therefore

$$
\ln \frac{y_{j}}{c_{j}}=\ln n
$$

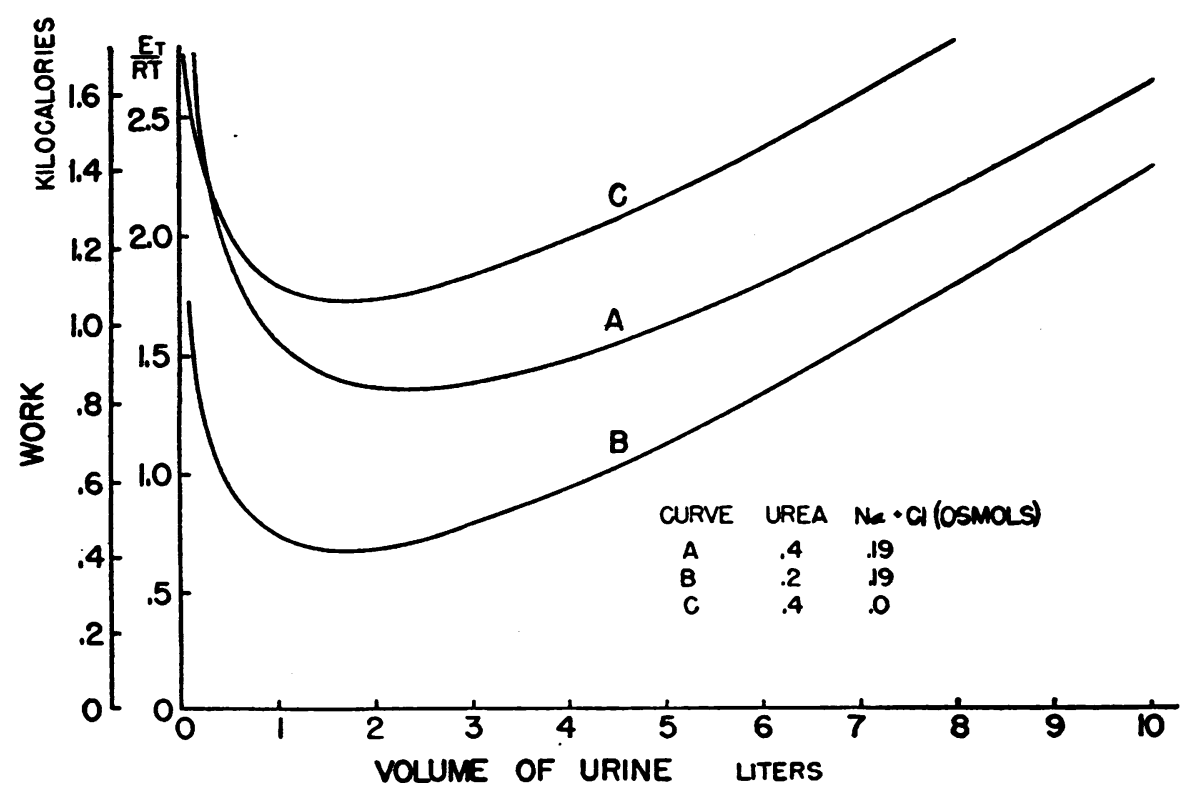

Fig. 1. The Amount of Osmotic Work Required for the Formation of Urine, Plotted as a Function of Total Daily Urine Volume

Curve $A$ is based on a typical normal urine of a subject on a high-protein diet. Curve $B$ is based on a urine containing only $1 / 2$ as much urea as that of curve $A$, but the same in other respects. Curve $C$ is calculated for a urine differing from that of curve $A$ in that it is $\mathrm{NaCl}$ free. 
or

$$
\frac{y_{j}}{c_{j}}=n \quad \text { and } \quad x_{j}=\frac{y_{j}}{n}=c_{j} .
$$

In the second case we have

$$
\begin{aligned}
\frac{\partial E_{T}}{\partial y_{j}}=R T & \left(y_{j} \frac{c_{j}}{y_{j}} \frac{1}{c_{j}}+\ln \frac{y_{j}}{c_{j}}\right. \\
& \left.-\ln \frac{\sum_{i} y_{i}}{\sum_{i} c_{i}}-\sum_{i} y_{i} \frac{\sum_{i} c_{i}}{\sum_{i} y_{i}} \frac{1}{\sum c_{i}}\right)=0,
\end{aligned}
$$

therefore

$$
\ln \frac{y_{j}}{c_{j}}=\ln \frac{\sum_{i} y_{i}}{\sum_{i} c_{j}}
$$

or

$$
\frac{y_{j}}{c_{j}}=\frac{\sum_{i} y_{j}}{\sum_{i} c_{i}}=n \quad \text { and again } \quad x_{j}=c_{j} .
$$

In both cases, we thus reach the obvious conclusion that $E_{T}$ is a minimum when the urinary concentration $x_{j}$ equals the concentration in the glomerular filtrate $c_{j}$. It may be stated more generally, that $E_{T}$ will be reduced if $x_{j}$ is changed in the direction which makes it more nearly equal to $c_{j}$.

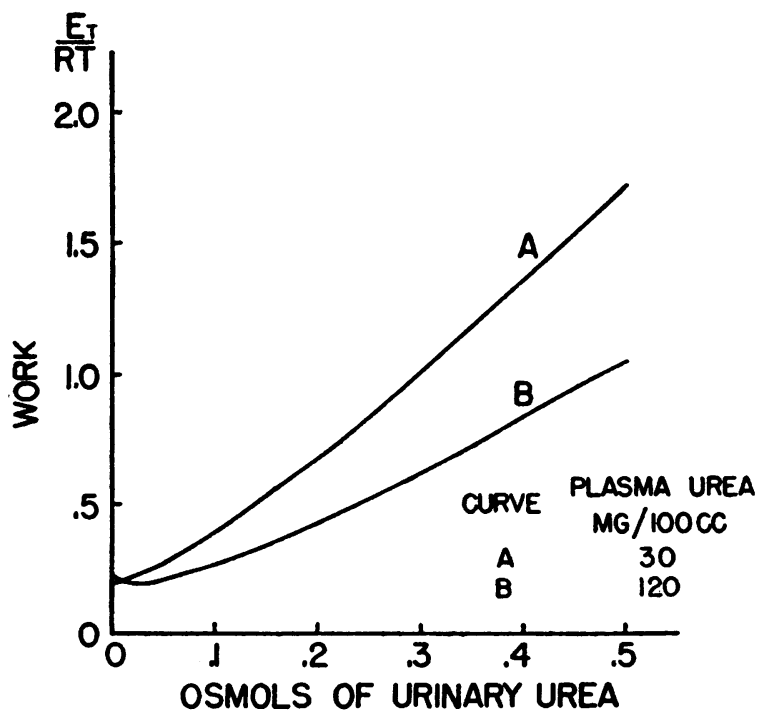

Fig. 2. Curves Which SHow How the Work is INFLUENCED BY THE AMOUNT OF UREA EXCRETED DAILY

For each point the amount of water has been adjusted so as to give minimal work (equation (5)). Curve $A$ is for a normal value of the plasma urea, while it is elevated to four times this value in curve $B$.
These results are important when applied to urea. Since urea is always greatly concentrated in the urine, a reduction in the amount of urea excreted will reduce the work. Curve A of Figure 1 is drawn for 0.4 osmols (24 grams) of urea. Curve $B$ represents a urine differing from that of curve A only in that it contains 0.2 osmols (12 grams) of urea per day. It is seen that a reduction of urea causes a significant reduction in the amount of work. In Figure 2, curve A also shows the effect on the energy of changing urinary urea. For each point, the amount of water is assumed to be that which will give the minimal amount of total work.

The experiments of Addis (2) show that rats, whose kidneys have been reduced to $1 / 4$ normal size, improve on a low-protein diet but become much worse on a high-protein diet. Since the rats on the high-protein diet excreted much more urea than the others, their kidneys did more osmotic work. This is interpreted as the basis for the marked difference in response of the two groups. ${ }^{4}$

When the generalization given above is applied to a substance less concentrated in the urine than in the glomerular filtrate, it follows that there will be a reduction in the work if the urinary concentration of the substance is increased. For example, the kidney must do work to prevent the excretion of glucose, and less work would be done if glucose were excreted so that the urinary and plasma concentrations were equal. Similarly, if the urinary concentration of $\mathrm{NaCl}$ is the same as the plasma concentration, no work is involved in its excretion, but if the dietary $\mathrm{NaCl}$ is limited, so that the urinary concentration is low, the kidney must do an increased amount of work. In Figure 1, curve $A$ is drawn for $\mathrm{Na}+\mathrm{Cl}=0.19$ mol per day, whereas curve $\mathrm{C}$ is the same, except $\mathrm{NaCl}$ is no longer being excreted. Curve $\mathrm{C}$ is seen to represent more work, especially for dilute urines.

Of the variables determining $E_{T}$ in equation (4), we have considered $n$ (total volume) and $y_{i}$ (amounts excreted): $c_{i}$ (plasma concentrations) remain to be considered. Examination of $\frac{\partial E_{T}}{\partial c_{j}}$ shows that as $c_{j}$ approaches the corresponding

\footnotetext{
4 It should be noted that some high-protein diets have a specific harmful effect on the kidneys (4).
} 
urinary concentration $x_{j}$, the work $E_{T}$ is reduced. For substance with $\frac{x_{j}}{c_{j}}$ less than 1 (e.g. glucose), decrease of $c_{j}$ will reduce the work, while if $\frac{x_{j}}{c_{j}}$ is greater than 1 , increasing $c_{j}$ will decrease the work. An increase in the blood urea above the normal value will cause a decrease in the work. In Figure 2, curve $A$ is drawn for a plasma urea of 0.005 osmols per liter $(30 \mathrm{mgm}$. urea per 100 cc.) and curve $B$ is for 0.020 osmols per liter (120 mgm. urea per $100 \mathrm{cc}$.). Curve B always is under curve A, except for the trivial case of urines with very low urea contents.

\section{THE RESULTS OF LIMITATION OF OSMOTIC WORK}

When the amount of osmotic work being done by the kidney is limited by the capabilities of the kidney, the results of this limitation may of ten be predicted from the equations for osmotic work. This limitation may occur in a normal kidney when an unusually large osmotic demand is made upon it, due, for example, to the restriction of drinking water. On the other hand, the kidney may be so injured that even the normal amount of work cannot be done. Thus, in severe chronic nephritis, many changes are observed which, as we shall see, may be referred to lack of ability to perform osmotic work. The result of limiting $E$ in the formulas for osmotic work is postulated to be the same as the changes seen in certain clinical conditions, notably severe chronic nephritis. This appears to be a reasonable postulate, and further support is lent it by the agreement of predictions based on it with fact.

It appears from Figure 1, or equation (4), that, for a urine containing any definite amount of solids per unit time, for each value of the work, there are two values of the volume $n$, corresponding to two concentrations. For values of $n$ between these two, the energy will be less, but for values of $n$ outside of this range, the energy will be greater. Thus, if the kidney is restricted to a certain maximum work, the concentration range of the urine is determined. As the maximum work decreases, the range of concentrations becomes narrower, so that for very restricted energies, the concentration has a very narrow range, corresponding to the minimal region of the curve. Since the minimum of the curve is at the point where the total osmolar concentration of the urine equals that of the glomerular filtrate (equation (5)), extreme limitation of the work implies an osmolar concentration of the urine not far from that of the glomerular filtrate, no matter what solids the urine contains.

In concentration-dilution tests, the range of possible concentrations is measured. As the urea clearance falls, the range of concentration becomes narrower (5). For extremely impaired renal function one would expect a specific gravity of about 1.010, corresponding to an osmolar concentration equal to that of the plasma. For very low urea clearances, the range of specific gravities is about 1.008 to 1.013 (from Alving and Van Slyke, Figure 3 (5)). These facts are in agreement with the theoretical predictions.

Besides these limitations, restrictions in the energy which can be expended with regard to single components is to be expected. The consequences of this limitation may be seen conveniently from equation (2). Figure 3 is a graph of this function, for $c=0.1$ and $c=0.2$. There is a zero value of the energy when $x=c$, the energy rising both for more dilute and more concentrated urines. For dilute urines, the slope of the curve is negative, so that the energy decreases with increasing concentration, while for urines concentrated with regard to the component under consideration, the energy increases with increasing concentration. There are thus two cases to be considered, one in which $x<c$, the other with $x>c$.

For a urine with a concentration greater than that of the glomerular filtrate with respect to a given component, a reduction in the energy must cause a lessened urinary excretion of the substance, and retention results. Retention will occur until a new blood concentration is reached, which is high enough so that the required amount of substance can be removed with the limited energy. For example, in Figure 3, normally 0.5 osmols per liter are removed by expenditure of energy $E=.405 R T$ calories per liter, with the normal plasma concentration of $c=0.1$. The possible energy expenditure is then reduced to $E=.158 R T$ and retention occurs because $x$ is reduced to 0.32 , until the plasma concentration reaches $c=0.2$. At this elevated plasma level, 


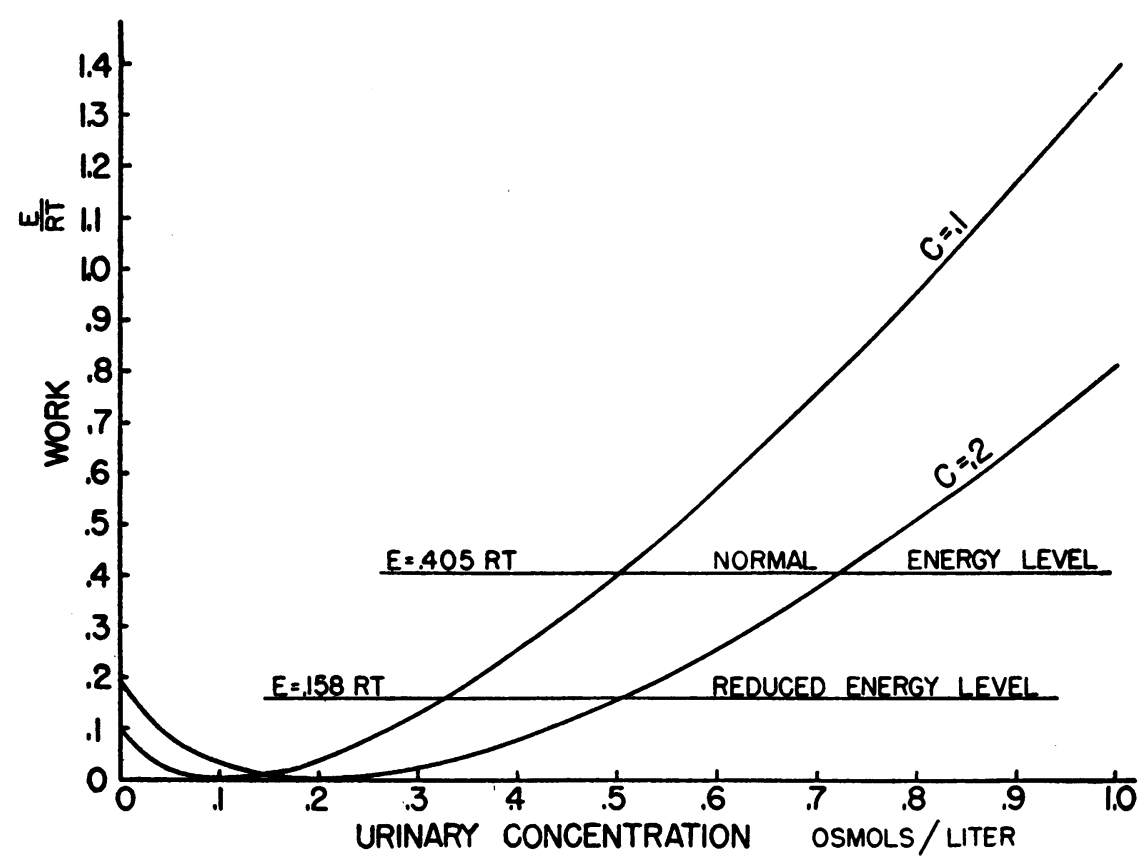

Fig. 3. Illustrating, for a Hypothetical Example, How Reduction of Renal Function Will Cause Progressive Retention Until the Plasma level Has Become High Enough So That, Even With the Reduced Ability to do Work, Sufficient Material Can be Excreted

It is also apparent that for a substance falling to the left of the minimum, reduction of renal function will cause an increased excretion of the substance.

the normal 0.5 osmols per liter can be removed with the limited energy, and no further retention occurs. A high blood urea may thus be considered both a result of a subnormal kidney, and a compensation for it.

Retention of urea, phosphate, and sulfate, all of which are greatly concentrated in the urine, occurs commonly in cases of kidney disease. Retention of urea occurs also after deprivation of water (6). Poisoning of the tubules with $\mathrm{NaCN}$ likewise causes reduced excretion of creatinine in dogs (7).

For a substance with respect to which the urine is relatively dilute, a reduction in the osmotic work necessitates an increase in urinary concentration, as may be seen from the left-hand part of the curves in Figure 3. Unless there is compensation for this increased excretion, the plasma level of the substance will fall until the rate of excretion is reduced to its normal value. Thus, when the intake of $\mathrm{NaCl}$ is not large, the plasma has a higher $\mathrm{NaCl}$ concentration than does the urine. Limitation of the osmotic work may thus result in an increased excretion of $\mathrm{NaCl}$, and a lowered plasma level.

In severe chronic nephritis, the expectation of a reduced plasma concentration of $\mathrm{Na}$, accompanied by an increase of plasma phosphate, sulfate, and urea, is actually realized (8). Also, kidneys poisoned with $\mathrm{NaCN}$ excrete more glucose and chloride, as shown in isolated dog kidneys (7).

If the energy expended on a substance is limited to a constant maximal value, and the substance is being excreted at a constant rate, it follows from equation $(3 a)$ that the ratio $\frac{x}{c}$ is constant. If the volume of urine is increased, there will be a temporary increase in the amount of substance excreted, until $c$ falls so that the ratio $\frac{x}{c}$ has reached its former value, and then $c$ will fall no more. This corresponds with the fact that on increasing the water drunk, the high blood urea of patients with chronic nephritis falls to a lower level, which is still above normal, and 
then remains constant at this lower level. The augmentation limit found in normal subjects may represent a similar, much higher, limitation on the energy expenditure.

For $\mathrm{NaCl}$, when its dietary intake is restricted, an increase in the urinary volume must either result in an increased expenditure of energy, or a reduction of the plasma level so that the previous $\frac{x}{c}$ ratio is re-established. If the energy is limited, a reduction of the blood $\mathrm{Na}^{+}$is to be expected. In the treatment of nephritic edema, removal of body $\mathrm{Na}$ is desired. A restricted intake of $\mathrm{Na}$ and a large urine volume would appear to work toward this end. The clinical effectiveness of this method has been shown (9). This procedure produces an increase in the osmotic demand made on the kidney, but is justified in this case because of its special therapeutic effect, the removal of edema. When edema is not an issue, there is no reason for not adjusting water and $\mathrm{NaCl}$ so as to give the minimal amount of work, and this means giving suitably large amounts of $\mathrm{NaCl}$.

Sweat is a solution containing a lower concentration of $\mathrm{NaCl}$ than the plasma. An increase in the rate of production of sweat at a given concentration will require an increased rate of work by the sweat glands. If this work cannot be done, the concentration of $\mathrm{NaCl}$ in the sweat must increase. It has been found that when sweating is very profuse, the concentration of $\mathrm{NaCl}$ is elevated (10).

Thus far we have considered only the work required for the idealized formation of urine. Although a satisfactory interpretation of many phenomena can be given on the basis of this simple idea, it would not be surprising if a more realistic consideration were sometimes required. The following example may be such a case, although an alternative interpretation is possible. Postulation of an upper limit to the rate at which the kidney can do osmotic work in reabsorbing glucose gives rise to a "threshold." But this cannot account for the fact that with high values of the blood glucose, the urinary concentration exceeds the blood concentration, since less osmotic work would be done if less glucose were excreted. This may result from the fact that, in order to make a substance pass through the tubular wall at a finite rate, work must be done, due to viscosity, aside from the osmotic work, due to a concentration difference. This effect is, of course, not taken into account in the equation for the reversible work. That glucosuria is due to the inability of the kidney to do osmotic work may be questioned. If another mechanism is involved, there would be no contradiction with even the simpler consideration.

In the case of substances which are more dilute in the urine than in the plasma, consideration of the work required for the diffusion through the tubular wall produces no contradiction, because limitation of either this work or the osmotic work requires the excretion of more of the substance. Furthermore, it may be inferred from the observed facts discussed above that in many cases the osmotic work is the determinative factor.

\section{SUMMARY}

The equation which gives the amount of work required for the idealized formation of urine has been examined in order to determine the amounts of excretory water and solids which will give the least work. Furthermore, the effect of changes in blood concentrations on osmotic work is determined. The changes necessitated by limitation in osmotic work are predicted and examples are given where these predictions agree with clinical findings. These considerations suggest certain therapeutic procedures, whose validity can be determined only by actual trial.

I am grateful to Doctors J. L. Gamble and L. H. Newburgh for encouragement throughout the preparation of this paper.

\section{BIBLIOGRAPHY}

1. von Rhorer, L., Über die osmotische Arbeit der Nieren. Arch. f. d. ges. Physiol., 1905, 109, 375.

2. Addis, T., The osmotic work of the kidney and the treatment of glomerular nephritis. Tr. A. Am. Physicians, 1940, 55, 223.

3. Borsook, H., and Winegarden, H. M., The work of the kidney in the production of urine. Proc. Nat. Acad. Sc., 1931, 17, 3.

4. Newburgh, L. H., and Johnston, M. W., High nitrogen diets and renal injury. J. Clin. Invest., 1931, 10, 153.

5. Alving, A. S., and Van Slyke, D. D., The significance of concentration and dilution tests ín Bright's disease. J. Clin. Invest., 1934, 13, 969.

6. Coller, F. A., and Maddock, W. G., Study of dehydration in humans. Ann. Surg., 1935, 102, 947. 
7. Bayliss, L. E., and Lundsgaard, E., The action of cyanide on the isolated mammalian kidney. $\mathrm{J}$. Physiol., 1932, 74, 279.

8. Gamble, J. L., Extracellular Fluid. Spaulding Moss Co., Boston, 1941. Chart 41.
9. Newburgh, L. H., and Lashmet, F. H., The importance of dealing quantitatively with water in the study of disease. Am. J. M. Sc., 1933, 186, 461.

10. Dill, D. B., Life, Heat, and Altitude. Harvard University Press, Cambridge, 1938. 